\title{
Fast Terminal Sliding Control Application for Second-Order Underactu- ated Systems
}

Ansu Man Singh* and Q. P. Ha

\begin{abstract}
In this paper, we propose a robust and finite-time control method, based on the terminal sliding mode (TSM), for a class of two-degree-of-freedom (2-DOF) underactuated electromechanical systems subject to bounded uncertainties and disturbances. First, the proposed Fast Terminal Sliding Mode (FTSM) method is presented. Then for the underactuated system control, hierarchical sliding surfaces are defined, consisting of two layers. In the first layer, separate FTSM sliding functions are selected for each state of the system. In the second layer, the system sliding manifold is a linear combination of the first layer sliding surfaces. A control law is derived and stability conditions of the nonlinear system are obtained by using the Lyapunov theory. To verify the effectiveness of our proposed method, the developed control technique is applied to control both the swinging load and the cart position of an underactuated gantry crane. Extensive simulation and real-time experiments demonstrate enhanced performance of the system and robustness against parametric variations in comparison to conventional TSM and sliding mode control.
\end{abstract}

Keywords: Fast Terminal Sliding Mode, Underactuated Systems, Hierarchical Sliding Mode, Swinging Load, Gantry Crane

\section{INTRODUCTION}

Control and estimation of underactuated uncertain systems, driven by fewer actuators than degrees of freedom, remain an interesting topic in control engineering [1-5]. Automated cranes with a swing load are quite popular among underactuated electromechanical systems and have been the subject of many studies [6]. In crane automation, underactuation, parametric variations and external disturbances, are quite often the reasons for difficulties in controlling the swinging load and trajectory following. For example, in [7], a flatness-based control method is presented for gantry cranes to achieve control goals in minimum transition time. Moreover, a sensing technique is developed for overhead cranes by using a vision sensor in [8], which can provide efficient visual feedback to the control the crane system in three-dimensional operations. For crane control, a considerable amount of literature has been published to address the problem of suppressing the swinging load vibration. For instance, a controller to minimise the oscillation of the payload due to parametric resonance is presented in [9].

To deal with system uncertainties, an adaptive method is proposed in [4] to control the hoisting/lowering operation of cranes' swinging load. Alternatively, problems of uncertainties in crane systems can be solved by employing robust controllers, for which the Sliding Mode Control
(SMC) method is very promising. Indeed, many published studies have shown the advantage of such controllers. For example, a sliding mode controller is presented in [10] for the trolley and load-hoisting dynamics where load-swing dynamics was coupled into the sliding surface for the trolley motion. The undesirable chattering in SMC signals can be reduced with a boundary layer or by using a higherorder sliding mode controller [11]. To this end, promising for mechanical and mechatronic systems are second-order sliding mode techniques, see e.g. [12,13]. In [14], secondorder sliding mode controllers are designed for container cranes in two phases, namely travelling and arrival phase. The modelling and trajectory tracking problem for the offshore cranes is addressed in [15] by using robust optimal sliding mode control. Techniques for adjusting control parameters have also been integrated into SMC for performance improvements. For example, in [16], a fuzzy disturbance estimator is coupled with the SMC technique to deal with the varying cable length in overhead cranes.

Studies on SMC of underactuated systems have mostly focused on the controllers that utilise linear sliding surfaces, e.g. [17] for container cranes, but do not directly address the problem of stability in finite time for underactuated systems subject to uncertainties. Requirements of finite-time stability can be fulfilled by designing sliding surfaces, based on Terminal Sliding Mode (TSM) [18]. The development of TSM has been applied to engineer-

Ansu Man Singh and Q.P. Ha are with School of Electrical and Data Engineering, University of Technology Sydney, Australia (e-mails: \{AnsuMan.Singh, Quang.Ha\}@uts.edu.au)

* Corresponding author 
ing systems such as robotic manipulators [19], permanent magnet synchronous motor [20], and hypersonic vehicles [12]. However, there are practical issues with performance, particularly in high-precision positioning applications, due to the slower response of TSM when the system's states are far from equilibrium. One way to address this problem is by combining the sliding surfaces of TSM and conventional SMC so that fast transient convergence both at a distance from and at a close range of the equilibrium can be obtained. The controller formed from such combination is known as Fast Terminal Sliding Mode Control (FTSM) as reported in [21]. In that work, the FTSM control law was proposed for a Single-input Single-output nonlinear system subject to the satisfaction of an applicability condition for the control design.

While theoretically promising, FTSM has found little application in real-time systems. So far, the practical implementation of FTSM control has been reported only for fully-actuated systems such as robotic manipulators [22]. To date, no studies have shown its application on underactuated systems such as an overhead crane with swinging load. To this end, we present in this paper the design and implementation of a type of finite-time, stable and robust FTSM controller for gantry cranes as a class of 2-DOF underactuated systems. First, after introducing the mathematical model of the system subject to uncertainties and disturbances that are assumed to be bounded, FTSM schemes are applied for the system states to achieve a shorter reaching time than the conventional TSM. Then, and a control law is derived, wherein sliding surfaces based on the Hierarchical Sliding Mode with two layers are proposed, using refined results from [23]. The first layer includes sliding functions based on FTSM to enable fast convergence to the system equilibrium, as required in high precision tracking applications. The second layer represents a linear combination of the first layer sliding functions. Stability of the closed loop system is analysed by using the Lyapunov Stability Theory. Extensive simulation and experimental results are presented to verify the advantage of the proposed control scheme.

\section{SYSTEM MODELLING AND FTSM}

\subsection{Mathematical model of underactuated systems}

Consider a class of 2-DOF underactuated mechanical systems described by the following Lagrangian equation

$$
\begin{aligned}
{\left[\begin{array}{ll}
m_{11}(\boldsymbol{q}) & m_{12}(\boldsymbol{q}) \\
m_{21}(\boldsymbol{q}) & m_{22}(\boldsymbol{q})
\end{array}\right]\left[\begin{array}{l}
\ddot{q}_{a} \\
\ddot{q}_{u}
\end{array}\right]+\left[\begin{array}{l}
C_{1}(\boldsymbol{q}, \dot{\boldsymbol{q}}) \\
C_{2}(\boldsymbol{q}, \dot{\boldsymbol{q}})
\end{array}\right] } \\
+\left[\begin{array}{l}
g_{1}(\boldsymbol{q}) \\
g_{2}(\boldsymbol{q})
\end{array}\right]=\left[\begin{array}{l}
\tau \\
0
\end{array}\right]+\boldsymbol{F}_{s}(\dot{\boldsymbol{q}})
\end{aligned}
$$

where $\boldsymbol{q}=\left[q_{a} q_{u}\right] \in \mathbb{R}^{2}, q_{a} \in \mathbb{R}$ and $q_{u} \in \mathbb{R}$ are respectively the actuated and underactuated generalized coordinates of the system,

$$
\left[\begin{array}{ll}
m_{11}(\boldsymbol{q}) & m_{12}(\boldsymbol{q}) \\
m_{21}(\boldsymbol{q}) & m_{22}(\boldsymbol{q})
\end{array}\right]=\boldsymbol{M}(\boldsymbol{q}) \in \mathbb{R}^{2 \times 2}
$$

is the inertial matrix which is assumed to be symmetric and positive definite, i.e. $\boldsymbol{M}(\boldsymbol{q})=\boldsymbol{M}(\boldsymbol{q})^{\top}$ and $\boldsymbol{M}(\boldsymbol{q})>$ 0. Similarly, $C_{1}(\boldsymbol{q}, \dot{\boldsymbol{q}})$ and $C_{2}(\boldsymbol{q}, \dot{\boldsymbol{q}})$ are the Coriolis and centrifugal terms, $g_{1}(\boldsymbol{q})$ and $g_{2}(\boldsymbol{q})$ are the gravitational terms, $\tau \in \mathbb{R}$ is the generalized input force or torque, and $\boldsymbol{F}_{s}(\dot{\boldsymbol{q}})$ is due to friction. In order to represent the dynamic and static behaviour of $\boldsymbol{F}_{S}(\dot{\boldsymbol{q}})$, we consider the following model [24, 25]: where $\boldsymbol{z}$ is the internal friction state, $\varphi(\dot{\boldsymbol{q}}) \in \mathbb{R}$ is a velocity dependent function, $\Psi(\dot{\boldsymbol{q}}) \in \mathbb{R}^{2}$ is a memoryless velocity-dependent function, $\boldsymbol{\Upsilon}_{0} \in \mathbb{R}^{2 \times 2}$ is a matrix of stiffness, and $\Upsilon_{1} \in \mathbb{R}^{2 \times 2}$ is a memoryless velocity-dependent matrix. Similarly, $\varphi(\dot{\boldsymbol{q}})$ can be represented by the following equation:

$$
\boldsymbol{\varphi}(\dot{\boldsymbol{q}})=F_{c}+\left(F_{\hat{s}}-F_{c}\right) \exp \left(\frac{\|\dot{\boldsymbol{q}}\|}{\dot{\boldsymbol{q}}_{s}}\right)^{2},
$$

where $F_{c}$ is Coulumb friction, $F_{\hat{s}}$ is the stiction friction, and $\dot{\boldsymbol{q}}_{s}$ is the Stribeck velocity. Similarly, $\|\cdot\|$ is the Euclidean norm of a vector.

Now, Equation (1) can also be formulated as

$$
\begin{aligned}
& \ddot{q}_{a}=f_{1}(\boldsymbol{q}, \dot{\boldsymbol{q}})+b_{1}(\boldsymbol{q}) u+\sigma_{1}(\boldsymbol{q}) \\
& \ddot{q}_{u}=f_{2}(\boldsymbol{q}, \dot{\boldsymbol{q}})+b_{2}(\boldsymbol{q}) u+\sigma_{2}(\boldsymbol{q}),
\end{aligned}
$$

where

$$
\begin{aligned}
& f_{1}(\boldsymbol{q}, \dot{\boldsymbol{q}})=-\left\{m_{11}^{\prime}(\boldsymbol{q})\left(C_{1}(\boldsymbol{q}, \dot{\boldsymbol{q}})+g_{1}(\boldsymbol{q})\right)\right. \\
&\left.+m_{12}^{\prime}(\boldsymbol{q})\left(C_{2}(\boldsymbol{q}, \dot{\boldsymbol{q}})+g_{2}(\boldsymbol{q})\right)\right\} \\
& f_{2}(\boldsymbol{q}, \dot{\boldsymbol{q}})=-\left\{m_{21}^{\prime}(\boldsymbol{q})\left(C_{1}(\boldsymbol{q}, \dot{\boldsymbol{q}})+g_{1}(\boldsymbol{q})\right)\right. \\
&\left.+m_{22}^{\prime}(\boldsymbol{q})\left(C_{2}(\boldsymbol{q}, \dot{\boldsymbol{q}})+g_{2}(\boldsymbol{q})\right)\right\}, \\
& {\left[\begin{array}{ll}
m_{11}^{\prime} & m_{12}^{\prime} \\
m_{21}^{\prime} & m_{22}^{\prime}
\end{array}\right]=M^{-1}(\boldsymbol{q}), } \\
& b_{1}(\boldsymbol{q})=m_{11}^{\prime}, \\
& b_{2}(\boldsymbol{q})=m_{21}^{\prime},
\end{aligned}
$$

and $u=\tau$ is the control input. In order to simplify control system design process, the terms $\sigma_{1}(\boldsymbol{q})$ and $\sigma_{2}(\boldsymbol{q})$ in Equation (3) are assumed to be uncertain, which represent the nonlinear friction model, i.e. Equation (2), and the unmodelled dynamics of the system. Furthermore, those functions are assumed to be bounded, i.e. $\left|\sigma_{1}(\boldsymbol{q})\right| \leq \mu_{1}$, and $\left|\sigma_{2}(\boldsymbol{q})\right| \leq \mu_{2}$.

\subsection{Fast Terminal Sliding Mode}

By adopting the idea of augmenting the terminal attractor [26], $\beta x^{q / p}$, with a linear attractor, $\alpha x$, the sliding function for the FTSM [21] is given by

$$
s=\dot{x}+\beta x^{q / p}+\alpha x
$$


where $\alpha>0, \beta>0, q>0$, and $p>0$, in which $q$ and $p$ are odd integers such that $q<p$ [27].

In the sliding mode, i.e. $s=0$, the state $x$ of equation (4) reaches the equilibrium point $x=0$ in a finite time which can be found in an explicit [21] form as,

$$
t_{F T S M}=\frac{p}{\alpha(p-q)} \ln \left(1+\frac{\alpha x_{0}^{(p-q) / p}}{\beta}\right)
$$

where $x_{0}=x(0)$. Moreover, the FTSM dynamics can be obtained by solving analytically the differential equation $\dot{x}+\beta x^{q / p}+\alpha x=0$, i.e. in the sliding mode $s=0$. Indeed, let's denote $z(x)=\alpha x^{(1-q / p)}+\beta$, one can derive:

$$
\begin{aligned}
t & =-\int_{z\left(x_{0}\right)}^{z(x)} \frac{d u}{u} \\
& =\frac{1}{\alpha(1-q / p)} \ln \left(\frac{\alpha x_{0}^{(1-q / p)}+\beta}{\alpha x^{(1-q / p)}+\beta}\right)
\end{aligned}
$$

and hence, the finite time $t_{F T S M}$ as in (4) can be obtained by letting $x=0$ in equation (5). An explicit form of the system state $x(t)$ in FTSM can also be obtained from (5) as given by [28]:

$$
x(t)= \begin{cases}\operatorname{sgn}\left(x_{0}\right) h(t) & t \leq t_{F T S M} \\ 0 & t>t_{F T S M}\end{cases}
$$

where,

$$
h(t)=\left(\frac{\left(\alpha x_{0}^{(p-q) / p}+\beta\right) e^{-\frac{\alpha(p-q)}{p} t}-\beta}{\alpha}\right)^{\frac{p}{p-q}} .
$$

The intuitive interpretation for the fast convergence of the sliding dynamics $s=0$ of FTSM is that when the state $x$ is far away from the equilibrium, the dynamics approximately becomes $\dot{x}=-\alpha x$, and then is reduced to $\dot{x}=-\beta x^{q / p}$ while close to $x=0$ [21]. This can be confirmed by an analytical comparison with the conventional Terminal Sliding Mode (TSM) of the following sliding function

$$
s=\dot{x}+\beta x^{q / p},
$$

which yields the system state

$$
x(t)= \begin{cases}\operatorname{sgn}\left(x_{0}\right)\left(x_{0}^{(p-q) / p}-\beta(1-q / p)\right)^{\frac{p}{p-q}} & t \leq t_{T S M} \\ 0 & t>t_{T S M},\end{cases}
$$

where the TSM reaching time from initial state $x_{0}$ to the equilibrium $x=0$ is found as

$$
t_{T S M}=\frac{1}{\beta\left(1-\frac{q}{p}\right)} x_{0}^{(1-q / p)} .
$$

Lemma 1: For the sliding functions of FTSM and TSM, represented by equations (4) and (8), respectively, if the sliding mode is achieved, i.e. $s=0$, then the time for FTSM to reach the equilibrium is less than that for TSM.

Proof: The lemma was first sketched in [21], here it is formulated with the following proof.

From the fact that $e^{\xi}>1+\xi, \forall \xi>0$, we have

$$
\exp \left(\frac{\alpha}{\beta} x_{0}^{(1-q / p)}\right)>1+\frac{\alpha}{\beta} x_{0}^{(1-q / p)} .
$$

Thus,

$$
\frac{\alpha}{\beta} x_{0}^{(1-q / p)}>\ln \left(1+\frac{\alpha}{\beta} x_{0}^{(1-q / p)}\right) .
$$

By multiplying both sides with $\frac{1}{\alpha\left(1-\frac{q}{p}\right)}$, we obtain:

$$
\frac{1}{\beta\left(1-\frac{q}{p}\right)} x_{0}^{(1-q / p)}>\frac{1}{\alpha\left(1-\frac{q}{p}\right)} \ln \left(1+\frac{\alpha}{\beta} x_{0}^{(1-q / p)}\right) .
$$

Hence,

$$
t_{T S M}>t_{F T S M}
$$

\section{FTSM FOR UNDERACTUATED SYSTEMS}

\subsection{Hierarchical sliding mode}

Let us first define the control error for a class of 2-DOF underactuated systems described in (3) as $e_{1}=q_{a}-q_{a d}$ and $e_{2}=q_{u}-q_{u d}$, where $q_{a d}$ and $q_{u d}$ are the desired references. Now consider the following sliding surfaces for each error:

$$
\begin{aligned}
& s_{1}=\dot{e}_{1}+\alpha_{1} e_{1} \\
& s_{2}=\dot{e}_{2}+\alpha_{2} e_{2},
\end{aligned}
$$

where $\alpha_{1}>0$ and $\alpha_{2}>0$, and define a new sliding variable, based on the Hierarchical Sliding Mode (HSM) coupling the two sliding variables:

$$
S=k_{1} s_{1}+k_{2} s_{2}
$$

where $k_{1}>0$ and $k_{2}>0$.

In the HSM framework, $s_{1}$ and $s_{2}$ are known as the first layer sliding functions and $S$ is the second layer sliding function. In this paper, the HSM method by [23] is adopted here. Although the technique has been inspiring for the control design of underactuated systems, see, e.g. 
[29], the proof provided in [23] contains some inconsistencies, as commented in $[30,31]$. Indeed, it remains challenging for the complex problem of stabilising underactuated systems, wherein, as indicated by [30], in Theorem 1 of [23] asymptotic stability can only be obtained for either sliding surface $s_{1}$ or $s_{2}$ of the first layer.

It should be noted that the HSM approach looks similar to the backstepping method, in which the whole system is decomposed recursively into subsystems until the stage comprising external inputs [32]. For each backstepping stage a control law is designed to cancel out cross-coupling terms via a suitable Lyapunov function. While variations in the system parameters may cause imperfect cancellation of cross-couplings particularly for a large number of backstepping stages, this "term explosion" problem could be avoided in HSM sliding functions constructed such that higher layer is the linear combination of lower layers.

\subsection{Control system design}

Now, from (3), the following FTSM sliding surfaces are proposed for the first layer:

$$
\begin{aligned}
& s_{1}=\dot{e}_{1}+\beta_{1} e_{1}^{q_{1} / p_{1}}+\alpha_{1} e_{1} \\
& s_{2}=\dot{e}_{2}+\beta_{2} e_{2}^{q_{2} / p_{2}}+\alpha_{2} e_{2},
\end{aligned}
$$

where the control parameters $p_{1}, q_{1}, p_{2}$, and $q_{2}$ are odd integers such that $\frac{q_{1}}{p_{1}}<1, \frac{q_{2}}{p_{2}}<1, \beta_{1}>0, \beta_{2}>0, \alpha_{1}>0$ and $\alpha_{2}>0$. Moreover, in the second layer the HSM sliding function (12) is chosen such that $0<k_{1} \neq k_{2}>0$ and also

$$
k_{1} b_{1}(\boldsymbol{q})+k_{2} b_{2}(\boldsymbol{q}) \neq 0 \quad \forall \boldsymbol{q} \in \mathbb{R}^{2},
$$

to avoid singularity.

The control law for the class of 2-DOF underactuated systems (3) of this paper is then proposed as:

$$
u=-\frac{1}{k_{1} b_{1}(\boldsymbol{q})+k_{2} b_{2}(\boldsymbol{q})}\left\{u_{1}+u_{2}+u_{3}\right\},
$$

where

$$
\begin{aligned}
& u_{1}=k_{1}\left(f_{1}(\boldsymbol{q}, \dot{\boldsymbol{q}})+\beta_{1} \frac{q_{1}}{p_{1}} e_{1}^{q_{1} / p_{1}-1} \dot{e}_{1}+\alpha_{1} e_{1}-\ddot{q}_{a d}\right) \\
& u_{2}=k_{2}\left(f_{2}(\boldsymbol{q}, \dot{\boldsymbol{q}})+\beta_{2} \frac{q_{2}}{p_{2}} e_{2}^{q_{2} / p_{2}-1} \dot{e}_{2}+\alpha_{2} e_{2}-\ddot{q}_{u d}\right) \\
& u_{3}=\eta \operatorname{sgn}(S)+K S,
\end{aligned}
$$

and where the control gains $K$ and $\eta$ are chosen such that $K>0$ and $\eta>D_{m}=\sup _{t \geq 0}\left|k_{1} \sigma_{1}(\boldsymbol{q})+k_{2} \sigma_{2}(\boldsymbol{q})\right|$. Here, $|\cdot|$ represents the absolute values.

The proposed control scheme is formulated in the following theorem.
Theorem 1: Consider the underactuated nonlinear system (3), and the sliding functions given in equations (12) and (13). The sliding function $S$ is asymptotically stable, i.e. $S \rightarrow 0$, if the control law (15), where $\eta>D_{m}$, is applied to the system.

Proof: For the stability of the proposed control input let us define a Lyapunov candidate function for the sliding surface $S$ as

$$
V=\frac{1}{2} S^{2}
$$

Taking time derivative one can get

$$
\dot{V}=S \dot{S}
$$

Let us consider the time derivative of the sliding function (12):

$$
\begin{aligned}
\dot{S}= & k_{1}\left(\ddot{e}_{1}+\beta_{1} \frac{q_{1}}{p_{1}} e_{1}^{q_{1} / p_{1}-1} \dot{e}_{1}+\alpha_{1} \dot{e}_{1}\right) \\
& +k_{2}\left(\ddot{e}_{2}+\beta_{2} \frac{q_{2}}{p_{2}} e_{2}^{q_{2} / p_{2}-1} \dot{e}_{2}+\alpha_{2} \dot{e}_{2}\right)
\end{aligned}
$$

or,

$$
\begin{aligned}
\dot{S}= & k_{1}\left(f_{1}(\boldsymbol{q}, \dot{\boldsymbol{q}})+\beta_{1} \frac{q_{1}}{p_{1}} e_{1}^{q_{1} / p_{1}-1} \dot{e}_{1}+\alpha_{1} \dot{e}_{1}-\ddot{q}_{a d}+\sigma_{1}(\boldsymbol{q})\right) \\
& +k_{2}\left(f_{2}(\boldsymbol{q}, \dot{\boldsymbol{q}})+\beta_{2} \frac{q_{2}}{p_{2}} e_{2}^{q_{2} / p_{2}-1} \dot{e}_{2}+\alpha_{2} \dot{e}_{2}-\ddot{q}_{u d}+\sigma_{2}(\boldsymbol{q})\right) \\
& +\left(k_{1} b_{1}(\boldsymbol{q})+k_{2} b_{2}(\boldsymbol{q})\right) u .
\end{aligned}
$$

Now by substituting the control law (15) into the expression of $\dot{S}$ and eventually into equation (17), and after some arrangements we find that

$$
\begin{aligned}
\dot{V} & =S \dot{S}=S\left\{k_{1} \sigma_{1}(\boldsymbol{q})+k_{2} \sigma_{2}(\boldsymbol{q})-\eta \operatorname{sgn}(S)-K S\right\} \\
& =-K S^{2}-\eta|S|+\left(k_{1} \sigma_{1}(\boldsymbol{q})+k_{2} \sigma_{2}(\boldsymbol{q})\right) S .
\end{aligned}
$$

Therefore, we have

$$
\dot{V}=S \dot{S} \leq-K S^{2}-\eta|S|+\left|k_{1} \sigma_{1}(\boldsymbol{q})+k_{2} \sigma_{2}(\boldsymbol{q})\right||S| .
$$

Now, let

$$
D_{m}=\sup _{t \geq 0}\left|k_{1} \sigma_{1}(\boldsymbol{q})+k_{2} \sigma_{2}(\boldsymbol{q})\right| .
$$

Then, if $\eta$ is chosen such that $\eta>D_{m}$ one has

$$
\dot{V}<-K S^{2}-\left(\eta-D_{m}\right)|S|<0 .
$$

Thus, by the Lyapunov stability theory $S$ is stable. Now, integrating both sides of (16), we can obtain

$$
\begin{aligned}
V & =\frac{1}{2} S^{2} \\
& =V(0)+\int_{0}^{t}\left[-K S^{2}-\eta|S|+\left(k_{1} \sigma_{1}(\boldsymbol{q})+k_{2} \sigma_{2}(\boldsymbol{q})\right) S\right] d \tau \\
& \leq V(0)-\int_{0}^{\infty}\left(K S^{2}+\left(\eta-D_{m}\right)|S|\right) d \tau<V(0)<\infty .
\end{aligned}
$$


or,

$$
\lim _{t \rightarrow \infty} \int_{t=0}^{\infty}\left(K S^{2}+\left(\eta-D_{m}\right)|S|\right) d \tau<\infty .
$$

Now, from the Barbarat lemma

$$
\lim _{t \rightarrow \infty}\left(K S^{2}+\left(\eta-D_{m}\right)|S|\right)=0 .
$$

In other words,

$$
\lim _{t \rightarrow \infty} S=0
$$

Hence, the higher layer sliding surface $S$ is asymptotically stable and bounded for the control input (15).

Now, let us consider a crane and its dynamics. An underactuated overhead crane is shown in Fig. 1(a). It consists of a cart of mass $m_{c}$, a hoisting mechanism through a rope of length $l$, and a payload of mass $m_{p}$. The position of the cart $(x)$ and the swing angle $(\theta)$ of the payload represent the generalized coordinates $q_{a}$ and $q_{u}$, respectively. Here, we assume that the payload consists of a point mass and the stiffness of the hoisting rope is negligible. As shown in Fig. 1(a), the cart is driven by a DC motor via a rack and pinion mechanism. It should be noted that all nonlinearities introduced due to the drive system here is treated as uncertainties as described in Equation (3). The dynamic equations for the crane system of the form (3) are obtained as:

$$
\begin{aligned}
f_{1}(\boldsymbol{q}, \dot{\boldsymbol{q}})= & \frac{-m_{p} l^{2}\left(\frac{k_{T} k_{b}}{R_{a} r^{2}}\right) \dot{x}+m_{p}^{2} l^{2} \sin \theta(l \dot{\theta}+g \cos \theta)}{\left(m_{p}+m_{c}\right) m_{p} l^{2}-m_{p}^{2} l^{2} \cos ^{2} \theta}, \\
f_{2}(\boldsymbol{q}, \dot{\boldsymbol{q}})= & \frac{m_{p}\left(\frac{k_{T} k_{b}}{R_{a} r}\right) l \dot{\theta} \cos \theta}{\left(m_{p}+m_{c}\right) m_{p} l^{2}-m_{p}^{2} l^{2} \cos ^{2} \theta} \\
& -\frac{m_{p} l \sin \theta\left\{\left(m_{p}+m_{c}\right) g+m_{p} l \cos \theta \dot{\theta}^{2}\right\}}{\left(m_{p}+m_{c}\right) m_{p} l^{2}-m_{p}^{2} l^{2} \cos ^{2} \theta}, \\
b_{1}(\boldsymbol{q})= & \frac{m_{p} l^{2} \frac{k_{T}}{R_{a} r}}{\left(m_{p}+m_{c}\right) m_{p} l^{2}-m_{p}^{2} l^{2} \cos ^{2} \theta}, \\
b_{2}(\boldsymbol{q})= & \frac{-m_{p} l \frac{k_{T}}{R_{a} r} \cos \theta}{\left(m_{p}+m_{c}\right) m_{p} l^{2}-m_{p}^{2} l^{2} \cos ^{2} \theta} .
\end{aligned}
$$

where $k_{T}$ is the motor torque constant, $k_{b}$ is its back emf constant, $R_{a}$ is the armature resistance, and $r$ is the radius of the shaft attached to the motor. Here, the position and swing angle errors required for the proposed control law (15) are defined as $e_{1}=x-x_{d}$ and $e_{2}=\theta$, respectively.

Remark: For generic underactuated systems, as noted by Park [27] and Ma [30], although $S$ is asymptotically stable under the HSM framework, it may not guarantee the simultaneous stability of $s_{1}$ and $s_{2}$. Nevertheless, for the crane system (25), we have formulated the following Corollary in order to demonstrate that the lower layer sliding functions are also stable.
Corollary 1: Consider the crane dynamics described by (25) where the control input (15) is applied. If the higher layer sliding function $S$ for the system is asymptotically stable, then lower layer sliding functions $s_{1}$ and $s_{2}$ are also asymptotically stable, i.e. $s_{1} \rightarrow 0$ and $s_{2} \rightarrow 0$.

Proof: We provide the proof by contradiction.

From Theorem 1 it is clear that $\lim _{t \rightarrow \infty} S=0$. Now, let us assume that $s_{1}, s_{2}, \dot{s}_{1}$, and $\dot{s}_{2}$ go to non-zero values. Similarly, let us assume that

$$
\lim _{t \rightarrow \infty} k_{1} s_{1}=-\lim _{t \rightarrow \infty} k_{2} s_{2}
$$

or

$$
\lim _{t \rightarrow \infty}\left(k_{1} s_{1}+k_{2} s_{2}\right)=0
$$

Now, since $\dot{S}$ is uniformly continuous, from Barbarat Lemma $\lim _{t \rightarrow \infty} \dot{S}=0$, which implies:

$$
\lim _{t \rightarrow \infty}\left(k_{1} \dot{s}_{1}+k_{2} \dot{s}_{2}\right)=0
$$

Now, applying time derivatives of $s_{1}$ and $s_{2}$ into (27) results in

$$
\begin{aligned}
& \lim _{t \rightarrow \infty} k_{1}\left\{\ddot{e}_{1}+\alpha_{1} \dot{e}_{1}+\beta_{1} \frac{q_{1}}{p_{1}} e_{1}^{\frac{q_{1}}{p_{1}}-1} \dot{e}_{1}\right\}+ \\
& \lim _{t \rightarrow \infty} k_{2}\left\{\ddot{e}_{2}+\alpha_{2} \dot{e}_{2}+\beta_{2} \frac{q_{2}}{p_{2}} e_{2}^{\frac{q_{2}}{p_{2}}-1} \dot{e}_{2}\right\}=0 .
\end{aligned}
$$

For crane system $e_{1}=x-x_{d}$ and $e_{2}=\theta$, which leads to

$$
\begin{aligned}
& \lim _{t \rightarrow \infty} k_{1}\left\{\ddot{x}-\ddot{x}_{d}+\alpha_{1} \dot{e}_{1}+\beta_{1} \frac{q_{1}}{p_{1}} e_{1}^{\frac{q_{1}}{p_{1}}-1} \dot{e}_{1}\right\} \\
& +\lim _{t \rightarrow \infty} k_{2}\left\{\ddot{\theta}+\alpha_{2} \dot{e}_{2}+\beta_{2} \frac{q_{2}}{p_{2}} e_{2}^{\frac{q_{2}}{p_{2}}-1} \dot{e}_{2}\right\}=0 .
\end{aligned}
$$

By substituting $\ddot{x}$ and $\ddot{\theta}$ from (3) into (28), and arranging the resultant terms, one gets

$$
\begin{array}{r}
\lim _{t \rightarrow \infty} k_{1}\left\{f_{1}(\boldsymbol{q}, \dot{\boldsymbol{q}})-\ddot{x}_{d}+\alpha_{1} \dot{e}_{1}+\beta_{1} \frac{q_{1}}{p_{1}} e_{1}^{\frac{q_{1}}{p_{1}}-1} \dot{e}_{1}+\sigma_{1}(\boldsymbol{q})\right\} \\
+\lim _{t \rightarrow \infty} k_{2}\left\{f_{1}(\boldsymbol{q}, \dot{\boldsymbol{q}})+\alpha_{2} \dot{e}_{2}+\beta_{2} \frac{q_{2}}{p_{2}} e_{2}^{\frac{q_{2}}{p_{2}}-1} \dot{e}_{2}+\sigma_{2}(\boldsymbol{q})\right\} \\
+\lim _{t \rightarrow \infty}\left(k_{1} b_{1}(\boldsymbol{q})+k_{2} b_{2}(\boldsymbol{q})\right) u=0 .
\end{array}
$$

Applying (15) into (29) and after some arrangement results in:

$$
\lim _{t \rightarrow \infty}\left(k_{1} \sigma_{1}(\boldsymbol{q})+k_{2} \sigma_{2}(\boldsymbol{q})\right)-\lim _{t \rightarrow \infty} K S-\lim _{t \rightarrow \infty} \eta \operatorname{sgn}(S)=0,
$$

Or

$$
\lim _{t \rightarrow \infty}\left(k_{1} \sigma_{1}(\boldsymbol{q})+k_{2} \sigma_{2}(\boldsymbol{q})-\eta \operatorname{sgn}(S)\right)=\lim _{t \rightarrow \infty} K S
$$


Now, let us consider the left hand side of (30), i.e.

$$
\lim _{t \rightarrow \infty}\left(k_{1} \sigma_{1}(\boldsymbol{q})+k_{2} \sigma_{2}(\boldsymbol{q})-\eta \operatorname{sgn}(S)\right) .
$$

Here, it should be noted that the term $k_{1} \sigma_{1}(\boldsymbol{q})+k_{2} \sigma_{2}(\boldsymbol{q})-$ $\eta \operatorname{sgn}(S)$ is undefined at $S=0$. Therefore, to analyse it, let us assume that $S$ approaches to origin from right side, i.e. $S \rightarrow 0^{+}$, then the limit leads to

$$
k_{1} \sigma_{1}(\boldsymbol{q})+k_{2} \sigma_{2}(\boldsymbol{q})-\eta .
$$

Since, $\eta>D_{m}=\operatorname{sub}\left|k_{1} \sigma_{1}(\boldsymbol{q})+k_{2} \sigma_{2}(\boldsymbol{q})\right|$, the term is less than zero, i.e. $k_{1} \sigma_{1}(\boldsymbol{q})+k_{2} \sigma_{2}(\boldsymbol{q})-\eta<0$.

Similarly, when $S$ approach from left side, i.e. $S \rightarrow 0^{-}$, the limit results in

$$
k_{1} \sigma_{1}(\boldsymbol{q})+k_{2} \sigma_{2}(\boldsymbol{q})+\eta>0 .
$$

Here, in both cases

$$
\lim _{t \rightarrow \infty}\left(k_{1} \sigma_{1}(\boldsymbol{q})+k_{2} \sigma_{2}(\boldsymbol{q})-\eta \operatorname{sgn}(S)\right) \neq 0,
$$

which implies $\lim _{t \rightarrow \infty} S \neq 0$. Now this contradicts the result of Theorem 1. Hence, our initial assumptions $s_{1} \neq 0$ and $s_{2} \neq 0$ are not correct. As a result,

$$
\begin{aligned}
& \lim _{t \rightarrow \infty} s_{1}=0 \\
& \lim _{t \rightarrow \infty} s_{2}=0 .
\end{aligned}
$$

This completes the proof.

The result obtained is also coincident with Qian and Yi in [33]. Furthermore, $s_{1}$ and $s_{2}$ are the FTSM based functions of $e_{1}$ and $e_{2}$, respectively (13), therefore, they also approach equilibrium asymptotically.

In order to reduce the chattering in the input, the signum function in equation (15) may be replaced by a saturation functions. Details on the stability of the system due to such functions are provided in [34].

\section{SIMULATION RESULTS}

\subsection{Laboratory testbed and control design}

The laboratory testbed is shown in Fig. 1 (b), whose system parameters, along with the parameters of the controller used in the simulation and experiments are provided in the Table 1. As mentioned earlier the parameters $q_{1}, p_{1}, q_{2}$, and $p_{2}$ should be odd integers such that $q_{1}<p_{1}$ and $q_{2}<p_{2}$. Therefore, the parameters were selected randomly so that it satisfies above mentioned conditions. However, to tune the parameters $k_{1}, k_{2}, \beta_{1}, \beta_{2}, \alpha_{1}$, and $\alpha_{2}$ we applied a Genetic Algorithm (GA) in the simulation environment. The GA is a search optimization technique based on natural selection. It starts with the initial population of chromosomes where each individual represents a solution to a problem. Performance of each chromosomes is evaluated using a fitness function. The algorithm has three main processes, namely Selection, Crossover and Mutation, which are evaluated in each generation. Finally, the algorithm terminates if the fitness value of the individuals has reached a user-defined threshold, or the maximum number of generations has reached. Details on the algorithm can be found in [35].

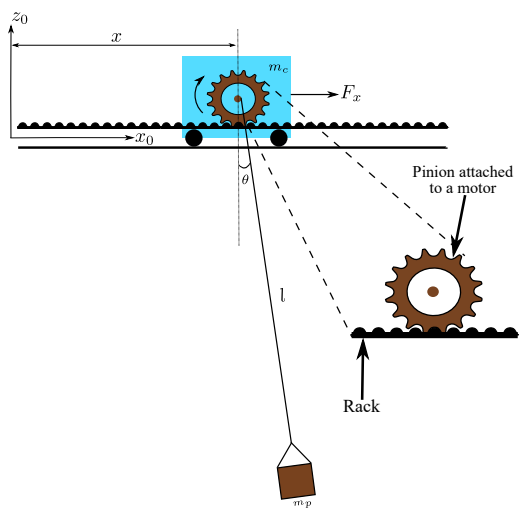

(a)

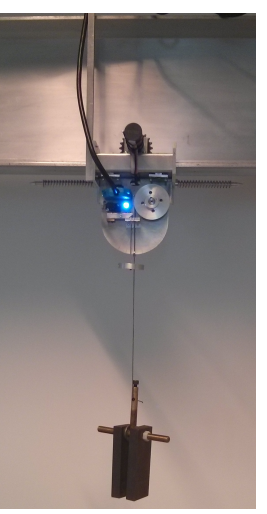

(b)
Fig. 1. Gantry crane: (a) diagram, and (b) laboratory testbed.

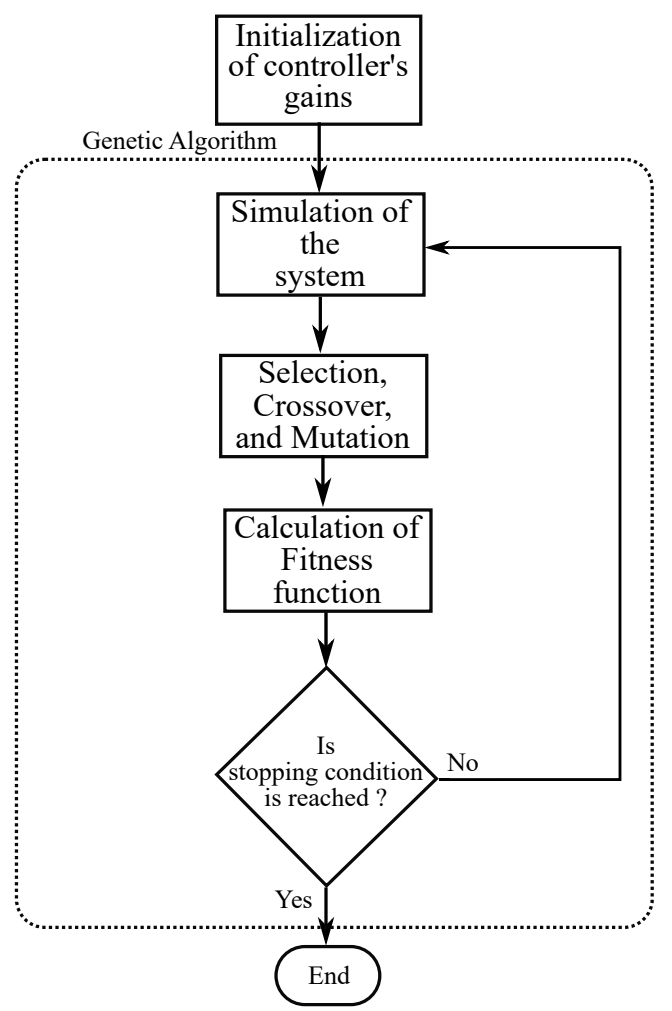

Fig. 2. Flowchart for tuning of controller gains using Genetic Algorithm 
Here the tuning process was conducted by using Matlab, and its flowchart is presented in Fig. 2. During the process, contents of each chromosomes of GA represents gain parameters of the controller which are randomly initialized. The performance of the chromosomes are evaluated by simulating the system, followed by calculation of the fitness function. We considered the Integral of Square Error (ITSE) as the fitness function, which is defined as:

$$
\text { ITSE }=0.5 \int_{0}^{t_{f}} t e_{x}^{2} d t+0.5 \int_{0}^{t_{f}} t \theta^{2} d t
$$

where $e_{x}=x-x_{d}$ and $t_{f}$ is the final time. After evaluation of the cost function, the selection, mutation and crossover operations of GA are applied. Finally, this whole process is repeated until a termination condition is reached. The gains after this tuning process are presented in Table. 1.

The control parameter $\eta$ is related to the convergence rate of the sliding mode, and its value should be greater than upperbounds of disturbances, i.e. $\mu_{1}$ and $\mu_{2}$. Nevertheless, higher values of the $\eta$ lead to the increment in chattering magnitude, whereas lower values decrease the robustness of the system. Therefore, the value of $\eta$ is manually adjusted during experiment.

\begin{tabular}{c|c}
\hline System parameter & Value \\
\hline$m_{p}$ & $2.24 \mathrm{~kg}$ \\
\hline$m_{c}$ & $2.7 \mathrm{~kg}$ \\
\hline$l$ & $1 \mathrm{~m}$ \\
\hline$g$ & $9.8065 \mathrm{~m} / \mathrm{s}^{2}$ \\
\hline$\mu_{1}$ & $0.1 \mathrm{~m} / \mathrm{s}^{2}$ \\
\hline$\mu_{2}$ & $0.1 \mathrm{rad} / \mathrm{s}^{2}$ \\
\hline \multirow{4}{*}{ Controller parameter } & $\alpha_{1}=1.75, \alpha_{2}=0.01, \eta=0.5$, \\
\cline { 2 - 2 } & $\beta_{1}=0.8, \beta_{2}=0.4$, \\
\cline { 2 - 2 } & $k_{1}=6.8, q_{2}=9, p_{2}=9, q_{2}=7$ \\
\hline
\end{tabular}

Table 1. System and control parameters

\subsection{Control performance}

Figure 3 (a) and (b) show simulation results of the position of the cart, the swing angle of the payload, and their velocities, respectively, for a cart position reference of $1.5 \mathrm{~m}$. The cart reaches its desired position in about 3.5 $\mathrm{sec}$ with a maximum velocity of $1 \mathrm{~m} / \mathrm{s}$. Furthermore, the swing of the payload diminishes at about the same-time with the peak oscillation of about $0.3 \mathrm{rad}$ (17.17 degrees). The angular velocity of the payload also dies out in aproximately 3 seconds.

The sliding functions and the control signal are depicted in Fig. 4 (a) and (b), respectively. Both first layer and second layer sliding functions, i.e. $s_{1}, s_{2}$, and $S$ are asymptotically convergent towards the equilibrium, $s_{1}=0, s_{2}=0$, and $S=0$. Similarly, the control input generated by the proposed technique is rather smooth with a maximum value of $8 \mathrm{~V}$.


(a)
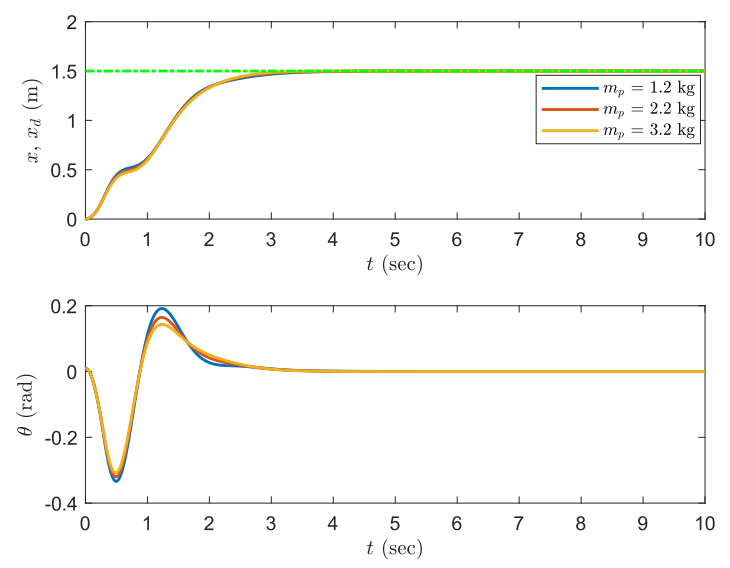

(b)

Fig. 5. Cart position and swing angle for variations in: (a) rope length, and (b) payload mass.

In order to test the robustness performance of the system under uncertainties, the system parameters, $l$ and $m_{p}$, are changed from their nominal values given in Table 1. Figure 5(a) shows the responses of the cart position $(x)$ and swing angle $(\theta)$ when the length of the rope varies by $20 \%$. From the figure, it can be observed that there is a small variation in the system response. For instance, the cart reaches its desired position in $3.5 \mathrm{sec}$, except when the length is $0.8 \mathrm{~m}$. Similarly, swing of the payload dies out in less than $5 \mathrm{sec}$ for all the cases. However, oscillation increases slightly and reaches the maximum value of $0.5 \mathrm{rad}$ (28.64 degrees) when the rope length is reduced to $0.8 \mathrm{~m}$ from the nominal value. Furthermore, the system shows little variation in performance with respect to the change in the mass of the payload. There are unnoticeable changes in trajectories of cart, and payload shows slight increment in peak oscillation when its mass is reduced to $1.2 \mathrm{~kg}$ (Fig. 5 (b)). These results clearly indicate that the proposed control law is immmune to the parametric un- 

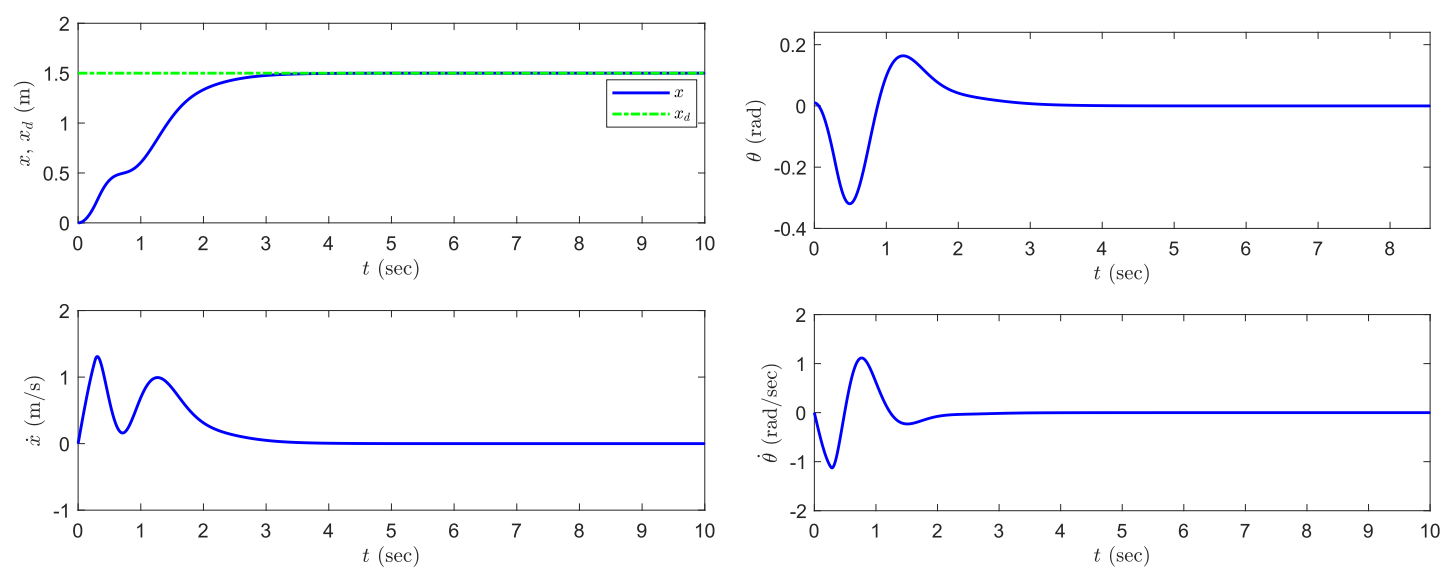

(a)

(b)

Fig. 3. Responses of (a) the trolley position, and (b) payload swing angle.



(a)

Fig. 4. Responses of (a) sliding functions $s_{1}, s_{2}$ and $S$, and (b) the control input $u$.

certainties.

The system was also evaluated under intrensic disturbances by adding the backlash or deadband into the input. The backlash nonlinearity considered during the simulation is given by [36]:

$$
D(u)= \begin{cases}\gamma_{1} u & u \geq \triangle \\ 0 & |u|<\triangle \\ \gamma_{2} u & u \leq-\triangle\end{cases}
$$

where $\gamma_{1}$ and $\gamma_{2}$ are the slope and $\triangle$ is the deadband. Figure 6 shows the response of the system for the backlash with deadband $(\triangle)$ of $0.5,1.0$, and $1.5 \mathrm{~V}$, respectively, and $\gamma_{1}=\gamma_{2}=1$. From the figure it can be observed that the transient response is similar to that of the previous cases, but there are some peak residual oscillations of $0.005 \mathrm{rad}$

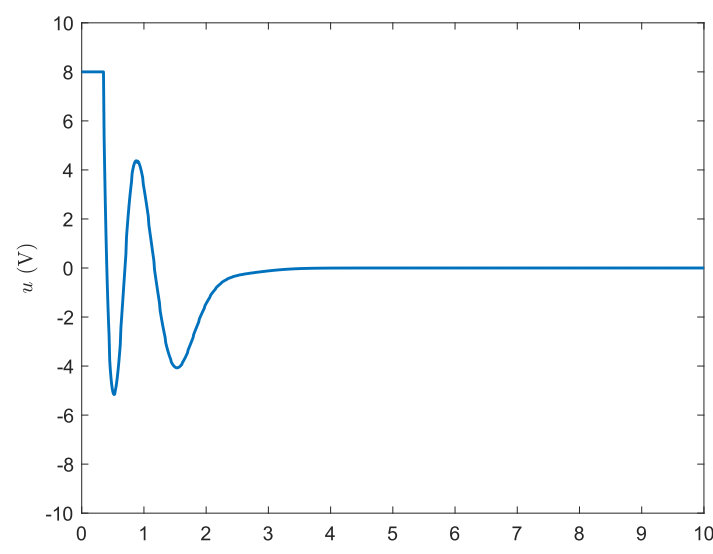

(b)

(0.25 degree) in the steady state condition, which can be observed in the zoomed section. Furthermore, the amplitude of the oscillation decreases with decreasing backlash voltage. Nevertheless, the residual swing is quite small. Therefore, it can be concluded that the system shows good performance under disturbances.

In Remark, it was concluded that $s_{1}$ and $s_{2}$ are asymptotically stable. However, in practice there always exist system nonlinearities in crane dynamics, such as mechanical hysteresis and backlash, which may affect the system performance. This is clear from the simulation result obtained by adding backlash nonlinearity, i.e. Fig. 6 . 

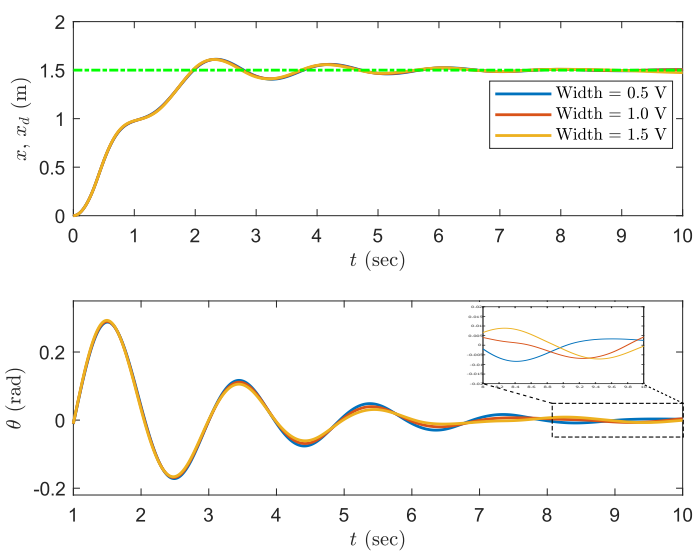

Fig. 6. Performance of the controller for different deadband.

As we mentioned earlier in Section 2.1, the disturbances such as backlash are modelled as $\sigma_{1}$ and $\sigma_{2}$ in the crane dynamics (3), which are assumed them to be bounded i.e. $\sigma_{1}<\mu_{1}$ and $\sigma_{2}<\mu_{2}$. However, in real systems, $\mu_{1}$ and $\mu_{2}$ are difficult to quantify because they are affected by various factors, such as unmodelled dynamics, friction, and so on. One of the solutions to the problem is to increase $\eta$, but as mentioned earlier in Section 4.1, it exacerbates the chattering as well. In this paper this parameter is adjusted manually so that the residual oscillation is minimum. This behaviour will also be discussed later while presenting the experimental results.

Now to show the advantage of the proposed approach, controllers designed from the Terminal Sliding Mode (TSM) and Sliding Mode Control (SMC) methods are compared with the FTSM controller within the same hierarchical sliding mode framework. The first layer sliding functions for TSM and SMC are defined respectively as in equations (37) and (38) below,

$$
\begin{aligned}
& s_{1}=\dot{e}_{1}+\beta_{1} e_{1}^{v_{1} / \rho_{1}} \\
& s_{2}=\dot{e}_{1}+\beta_{2} e_{2}^{v_{2} / \rho_{2}},
\end{aligned}
$$

and

$$
\begin{aligned}
& s_{1}=\dot{e}_{1}+\alpha_{1} e_{1} \\
& s_{2}=\dot{e}_{2}+\alpha_{2} e_{2} .
\end{aligned}
$$
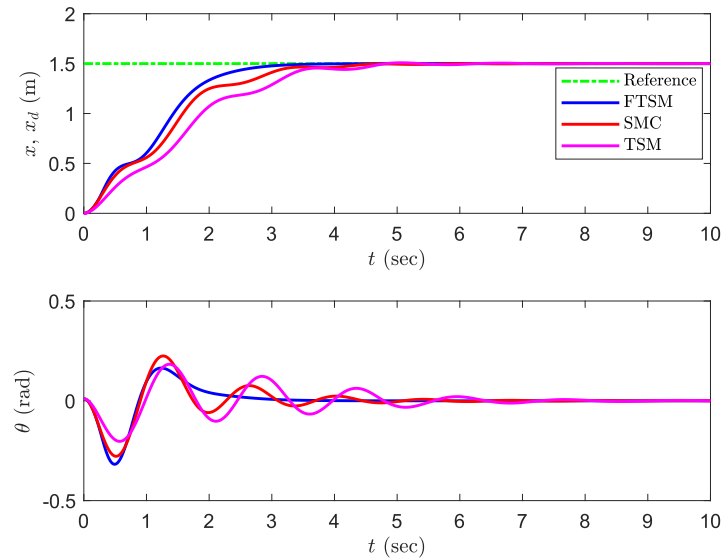

Fig. 7. Comparison of FTSM, TSM, and SMC responses for the cart position $x$, and payload swing angle $\theta$.

The overall control inputs $u$ for both controllers are similar to equation (15) but $u_{1}, u_{2}$ and $u_{3}$ are defined as

$$
\begin{aligned}
& u_{1}=k_{1}\left(f_{1}(\boldsymbol{q}, \dot{\boldsymbol{q}})+\beta_{1} e_{1}^{v_{1} / \rho_{1}-1} \dot{e}_{1}-\ddot{q}_{a d}\right) \\
& u_{2}=k_{2}\left(f_{2}(\boldsymbol{q}, \dot{\boldsymbol{q}})+\beta_{2} e_{2}^{v_{2} / \rho_{2}-1} \dot{e}_{2}\right) \\
& u_{3}=\eta \operatorname{sgn}(S)+K S,
\end{aligned}
$$

for TSM, while for SMC they are defined as

$$
\begin{aligned}
& u_{1}=k_{1}\left(f_{1}(\boldsymbol{q}, \dot{\boldsymbol{q}})+\alpha_{1} \dot{e}_{1}-\ddot{q}_{a d}\right) \\
& u_{2}=k_{2}\left(f_{2}(\boldsymbol{q}, \dot{\boldsymbol{q}})+\alpha_{2} \dot{e}_{2}\right) \\
& u_{3}=\eta \operatorname{sgn}(S)+K S,
\end{aligned}
$$

where $q_{a d}(t)$ is the desired trajectory of the trolley.

The TSM controller parameters $v_{1}, v_{2}, \rho_{1}, \rho_{2}$ should be odd integers such that $v_{1}<\rho_{1}$ and $v_{2}<\rho_{2}$. During the experiment, we used the same parameters that was applied in the proposed controller. However, to tune parameters $k_{1}, k_{2}, \alpha_{1}, \alpha_{2}, \beta_{1}$ and $\beta_{2}$, we applied the Genetic Algorithm as described in Section 4.1. The tuned parameters are presented in Table 2. For $\eta$, we used the same value as in the proposed controller case.

\begin{tabular}{c|c}
\hline Controller & Parameters \\
\hline \multirow{2}{*}{ TSM } & $\beta_{1}=0.7, v_{1}=9, \rho_{1}=11, k_{1}=9.5$ \\
\cline { 2 - 2 } & $\beta_{2}=0.03, v_{1}=7, \rho_{1}=9, k_{2}=4.4$ \\
\hline SMC & $\alpha_{1}=1.45, \alpha_{2}=0.05, k_{1}=4.2, k_{2}=2.9$ \\
\hline & $\eta=0.5, K=1$ \\
\hline
\end{tabular}

Table 2. Controller parameters for TSM and SMC

Results of the comparison for the cart position $(x)$ and the swing angle $(\theta)$ are presented in Fig. 7. The responses obtained clearly confirm the improved performance of the 
proposed controller regarding the settling time. For instance, the cart takes about 3.5 seconds to reach the reference position, compared to 4.5 seconds for TSM and about 5 seconds for SMC, respectively. Furthermore, swing angle of the payload reaches the equilibrium, i.e. $\theta=0$, in about 3 seconds with smooth trajectory, compared to SMC and TSM, which takes around 4 and 6 seconds, respectively. These results indicates the advantage of the proposed method over the other methods.

\section{EXPERIMENTAL RESULTS}

\subsection{Control implementation}

The set-up is depicted in Fig. 8 hich overhead crane, as shown in Fig. 1, a control board, signal conditioning circuits, and a Stm32f411 based microcontroller board. The crane consists of potentiometers for the measurement of the cart's position $(x)$ and the swing angle of the payload $(\theta)$, which can be described by the following equations:

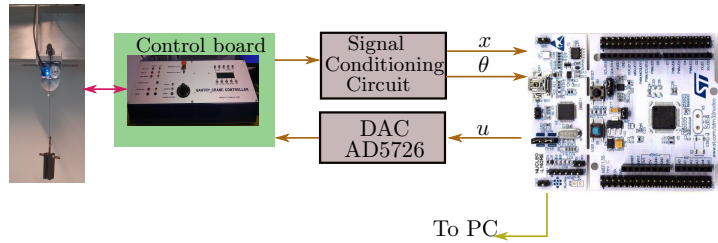

Fig. 8. Block diagram for implementation.

$$
\begin{aligned}
& y_{\theta}=\varsigma_{1} \theta+\varsigma_{2} \\
& y_{x}=\hat{\varsigma}_{1} x+\hat{\varsigma}_{2},
\end{aligned}
$$

where $y_{\theta}$ and $y_{x}$ are the measured quantities, $\varsigma_{1}$ and $\hat{\zeta}_{1}$ are the gains, and $\varsigma_{2}$ and $\hat{\zeta}_{2}$ are the offset in measurements. The parameters $\zeta_{1}, \hat{\zeta}_{1}, \zeta_{2}$ and $\hat{\zeta}_{2}$ are estimated by applying least-square curve fitting on the measured input and output datas. In addition, the sensors are connected to the control board which provides the electrical interface. Similarly, the proposed control algorithm is implemented in the microcontroller which consists of a ARM Cortex M4 processor with $512 \mathrm{~KB}$ of flash memory and $2 \mathrm{~KB}$ of RAM. It receives the feedback signals, i.e. $x$ and $\theta$ from the control board through a signal conditioning circuit, whose purpose is for an interface between the crane and the microprocessor. The control signal generated by the board is fed to crane through an AD5726 DAC IC. To collect crane sensors and control data for post-processing, a PC with the microprocessor board is interfaced via a UART protocol.
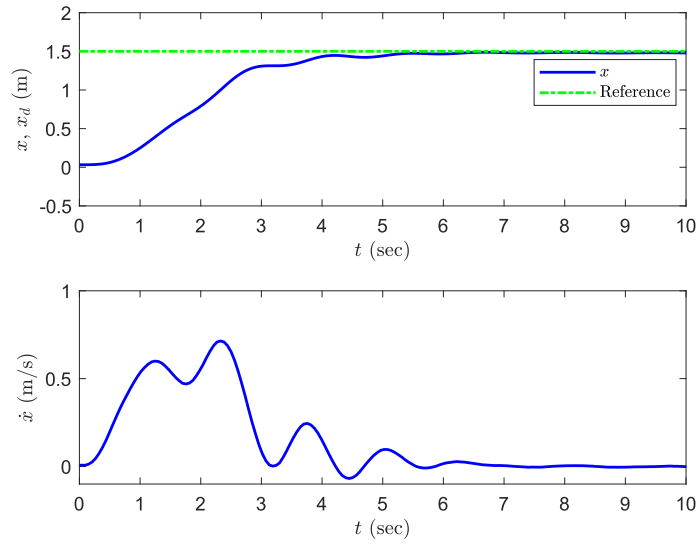

Fig. 9. Test results of the proposed method for cart position

The control input (Equation (15)) requires both position and the velocity of the feedback signals. Nevertheless, the velocities are not available from the system. Therefore, the signals are estimated using an approximate differentiator, which is defined as $\dot{\boldsymbol{q}}=\frac{1}{T}\left(\boldsymbol{q}(t)-\boldsymbol{q}\left(t-T_{S}\right)\right)$, where $T_{s}$ is the sampling period of the controller.

The Nucleo board runs FreeRTOS operating system (OS) and the proposed controller runs on the board as an OS's stack. The sampling time for the control system is 1 ms. Digital low pass filters are used to reduce the noise in the measurement.

\subsection{Test results}
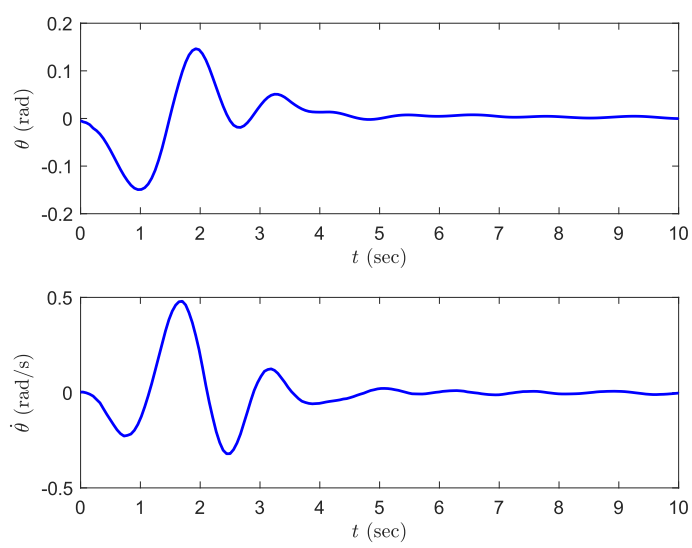

Fig. 10. Test results of the proposed method for swing angle.

In the experiments, the system and controller parameters are used as listed in Table 1 . The experimental response of the cart position $x$ is shown in Fig. 9 with a the desired position in 5 seconds. Similarly, the velocity of the cart $\dot{x}$ achieves the maximum value $0.75 \mathrm{~m} / \mathrm{s}$ and reaches the equilibrium eventually. Moreover, as shown in Fig. 
10 , the controller is able to suppress the payload swing $(\theta)$ and its angular velocity $(\dot{\theta})$. The maximum angle of the payload motion is about $0.15 \mathrm{rad}$ or 8.52 degrees. Furthermore, the system shows much lower residual oscillation of around 0.25 degrees. As mentioned earlier in Section 4.2, the residual oscillation is due to the disturbances such as backlash. To verify this, a comparison of the simulation results and experimental results is presented in Fig. 11 (a). The figure clearly indicates that the magnitude of residual oscillations, as observed in the zoomed section, are in the same range. Furthermore, the figure also shows that the response of the system is similar to that of simulation. For instance, the settling time for of the cart for both cases is around 5 seconds. There is, however, some differences in the transient response of simulation and the real-time experimental results, which is due to unmodelled nonlinearities.
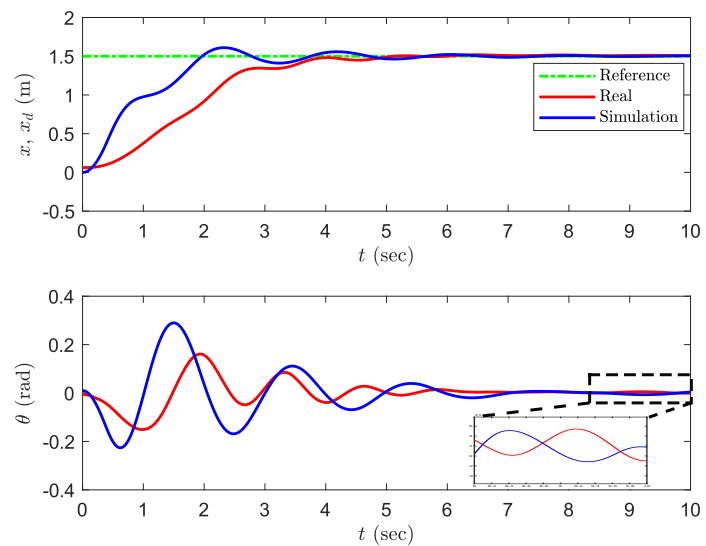

(a)


(b)

Fig. 11. Feasibility and performance of the proposed method: (a) comparison between simulation and experiment, and (b) sliding functions and control input.

Overall, the results clearly indicate that the proposed control method can stabilise the system and outperform the conventional SMC and TSM in terms of its fast response. Feasibility of the proposed control scheme is also verified as shown in Fig. 11(b) for the responses of the control signal and sliding functions.

It can be noticed that in Fig 11(b) the transient response of control input is not smooth. One of the reasons for such behaviour of the system is due to the unmodeled nonlinearities such as Equation (2) which are dependent on velocity.
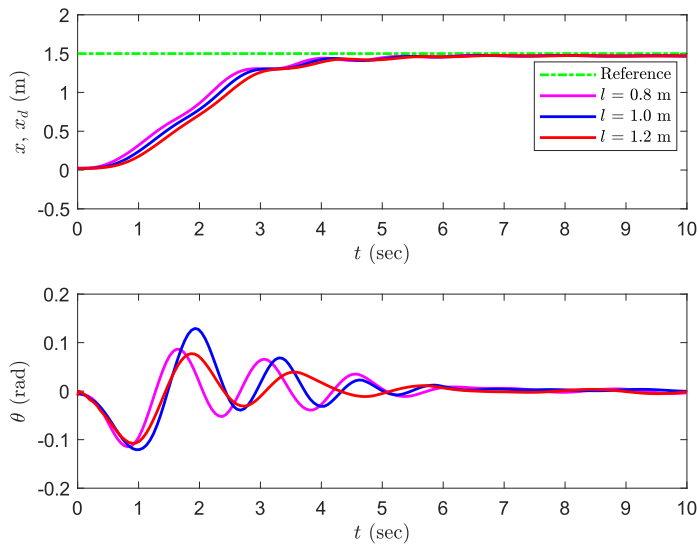

(a)


(b)

Fig. 12. Experimental responses of the cart position and swing angle: (a) robustness against parameter variations (b) comparison with TSM and SMC.

To judge robustness of the proposed control method, we created scenarios similar to those of simulation. For that we tested the crane performance by varying the length of the rope by $20 \%$ from the nominal value, i.e. $1 \mathrm{~m}$. Responses of the cart position and the swing angle are shown in Fig. 12(a). The experimental results clearly indicate the insensitivity of the proposed control system with respect to parameter variations. The results observed further coincide with those obtained from simulation. 
For comparison with TSM and SMC, the control laws as mentioned in Section 4.2 with the same design parameters. The time responses of the crane's cart position and payload angular swing are depicted in Fig. 12(b). Results of the experiment clearly show the advantage of our method regarding the convergence time. For instance, the cart takes about $5.0 \mathrm{sec}$ to reach the goal location in case of the proposed FTSM as compared to $5.5 \mathrm{sec}$ for TSM, and $6.0 \mathrm{sec}$ for SMC. The observations further verify the results obtained from simulation, as described in the previous section.

\subsection{Trajectory tracking}

\begin{tabular}{c|c}
\hline Parameters & Value \\
\hline$a$ & $0.2857 \mathrm{~m} / \mathrm{sec}^{2}$ \\
\hline$t_{1}$ & $1.5 \mathrm{sec}$ \\
\hline$t_{2}$ & $3.5 \mathrm{sec}$ \\
\hline$t_{F}$ & $5 \mathrm{sec}$ \\
\hline$T_{s}$ & $1 \times 10^{-3} \mathrm{sec}$ \\
\hline
\end{tabular}

Table 3. Reference trajectory parameters

To further experimentally evaluate the control performance, we apply a trapezoidal reference for the cart velocity. The trajectory consists of three phases, namely, acceleration, constant velocity and deceleration. The desired velocity $\left(\dot{x}_{d}\right)$ and position $x_{d}$ using the trapezoidal trajectory for the cart velocity can be described as:

$$
\dot{x}_{d}= \begin{cases}a t & 0<t \leq t_{1} \\ a t_{1} & t_{1}<t \leq t_{2} \\ a t_{1}-a\left(t-t_{2}\right) & t_{2}<t \leq t_{F},\end{cases}
$$

and

$$
x_{d}(t)= \begin{cases}a \frac{t^{2}}{2} & 0<t \leq t_{1} \\ a \frac{t_{1}^{2}}{2}+a t_{1}\left(t-t_{1}\right) & t_{1} \leq t \leq t_{2} \\ a \frac{t_{1}^{2}}{2}+a t_{1}\left(t_{2}-t_{1}\right)-\frac{a}{2}\left(t^{2}-t_{2}^{2}\right) & t_{2}<t \leq t_{F} .\end{cases}
$$

where $a$ is the acceleration, $t_{1}$ is the time instant indicating the end of the acceleration phase, $t_{2}$ is the time instant at the beginning of the deceleration phase, and $t_{F}$ is the final time period. The parameters of the reference trajectory for the experiment are shown in Table 2 . The sampling period $\left(T_{s}\right)$ of the controller is $1 \mathrm{~ms}$, much smaller than the motor time constant.
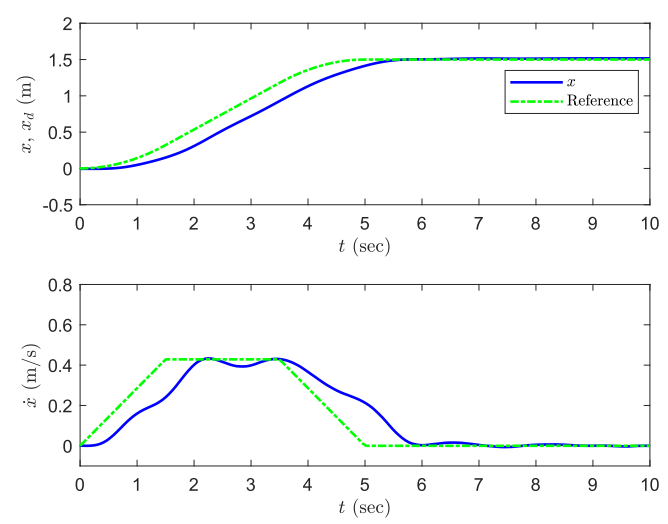

(a)
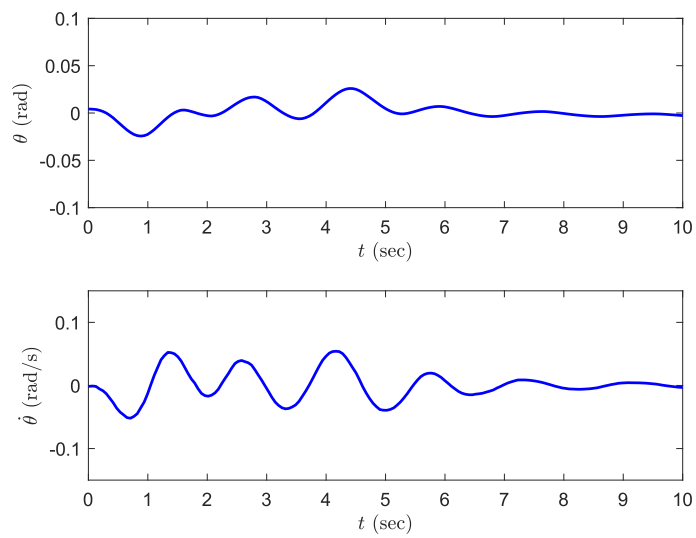

(b)

Fig. 13. Position and velocity trajectories of (a) the cart, and (b) swinging load.

Tracking performance of the proposed controller is presented in the Fig. 13, wherein it is clear that the controller is able to track the reference position and reach the final desired location in about $5 \mathrm{sec}$. Payload swing angle and its velocity, as shown in the Fig. 13 (b), are less than 3 degrees and 30 (degrees/sec), respectively. To indicate feasibility of the proposed FTSM controller, Fig. 14 depicts the sliding surfaces and control input of the controller. The control voltage signal during tracking the trapezoidal velocity reference is between -2 and $4 \mathrm{~V}$.

Robustness of the controller is again tested by varying the rope length by $20 \%$ from its nominal value, as can be seen in Fig. 15 (a) and (b), wherein the control performance of the position and velocity trajectories of the cart as well as the swinging load appears not to be effected by the length variations under the proposed controller.

The merit of the proposed controller is also confirmed by comparison with the Terminal Sliding Mode (TSM) and Sliding Mode Control (SMC). As shown in Fig. 16, the cart's position with FTSM is the closest to the reference trajectory.

The experimental results can also be evaluated in terms 


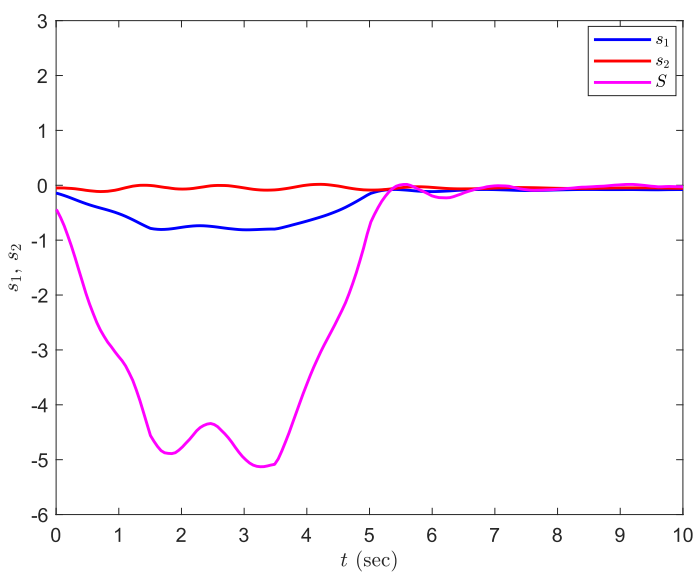

(a)

Fig. 14. Sliding surfaces and control input during tracking.
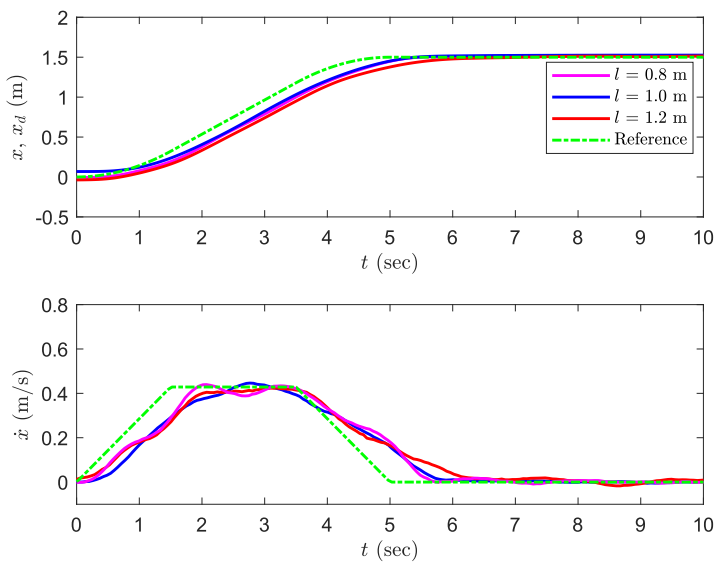

(a)


(b)



(b)

Fig. 15. (a) Cart position tracking and (b) swing angle for the proposed controller with varying rope length.

of performance indices such as the Integral of Squared Error (ISE) and Integral of Time Squared Error (ITSE). The results are summarised in the Fig. 17, where it can be seen that the tracking performance of the cart's position $(x)$ with FTSM is much better than with TSM or SMC judging by these indices.

\section{CONCLUSION}

In this paper, we have presented the design and implementation of a fast terminal sliding mode (FTSM) controller for a class of 2-DOF underactuated systems. The proposed method is based on a hierarchy of the first layer for FTSM control of each system variable and the second layer with a linear combination of the FTSM sliding func- 

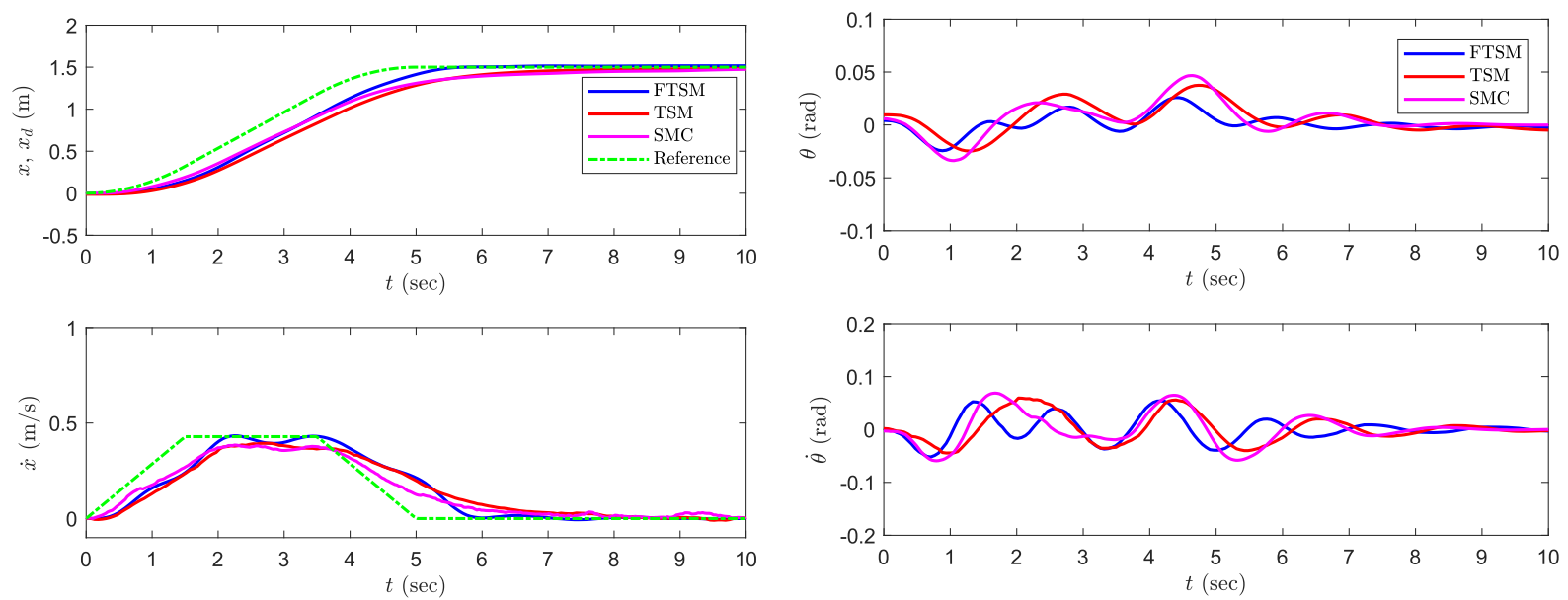

(a)

(b)

Fig. 16. Comparison of the proposed controller with TSM and SMC.
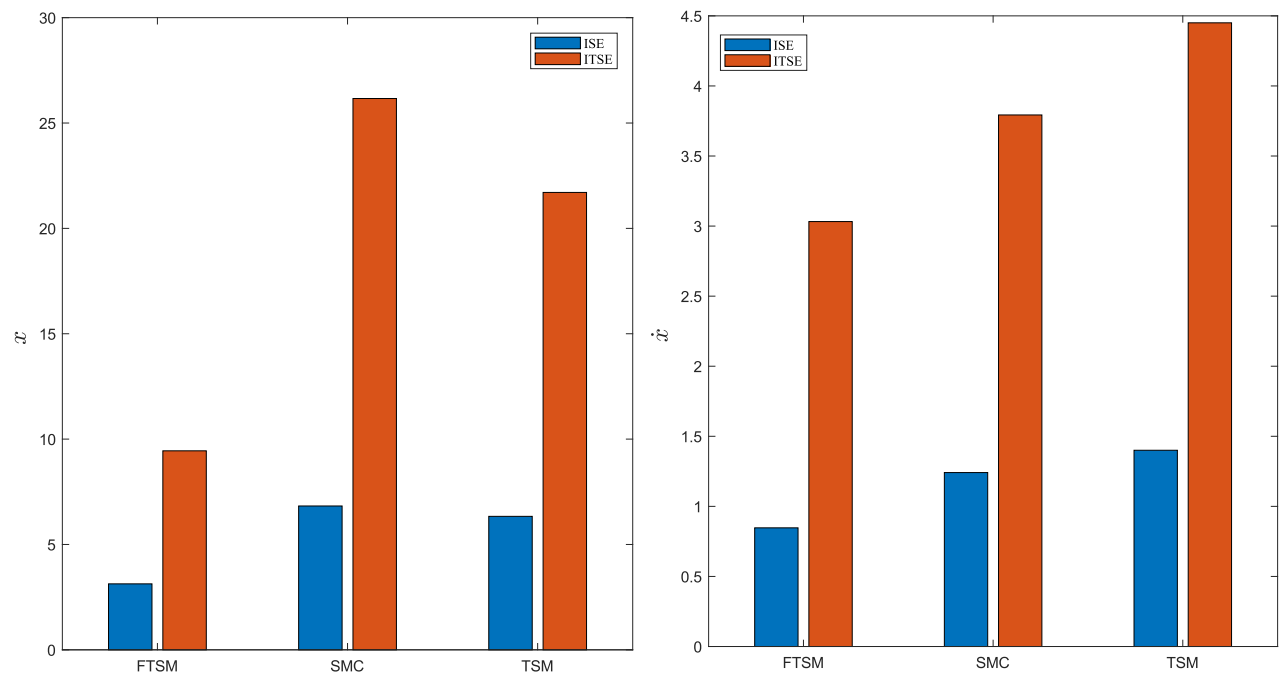

(a)

(b)

Fig. 17. ISE and ITSE errors for a) cart's position and b) velocity tracking.

\section{REFERENCES}

[1] M. Mirzaei, N. Meskin, and F. Abdollahi, "Robust consensus of autonomous underactuated surface vessels," IET Control Theory and Applications, vol. 11, no. 4, pp. 486494, 2017. doi: 10.1049/iet-cta.2016.0930.

[2] J. Davila, L. Fridman, and A. Levant, "Second-Order Sliding-Mode Observer for Mechanical Systems," IEEE Trans. Automat. Contr., vol. 50, no. 11, pp. 1785-1789, 2005. doi: 10.1109/TAC.2005.858636.

[3] S. H. Lee, J. B. Park, and Y. H. Choi, "Finite time control of nonlinear underactuated systems using terminal sliding surface," IEEE International Symposium on Industrial Electronics, pp. 626-631, 2009 . doi: 10.1109/ISIE.2009.5220307.

[4] N. Sun, Y. Fang, H. Chen, and B. He, "Adaptive nonlinear crane control with load hoisting/lowering and unknown parameters: design and experiments," IEEE/ASME Transactions on Mechatronics, vol. 20, no. 5, pp. 2107 - 2119, 2015. doi: 10.1109/TMECH.2014.2364308.

[5] D. Zehar, K. Benmahammed, and K. Behih, "Control for Underactuated Systems Using Sliding Mode Observer," International Journal of Control, Automation and Systems, vol. 16, pp. 739-748, apr 2018. doi: 10.1007/s12555-0160730-2. 
[6] E. Abdel-Rahman and A. Nayfeh, "Dynamics and control of cranes: A review," Journal of Vibration and Control, vol. 9, no. 7, pp. 863-908, 2003. doi: 10.1177/2F1077546303009007007.

[7] B. Kolar, H. Rams, and K. Schlacher, "Time-optimal flatness based control of a gantry crane," Control Engineering Practice, vol. 60, no. Supplement C, pp. 18-27, 2017. doi: 10.1016/j.conengprac.2016.11.008.

[8] L. Lee, C. Huang, S. Ku, and Z. Yang, "Efficient visual feedback method to control a three-dimensional overhead crane," IEEE Transactions on Industrial Electronics, vol. 61, no. 8, pp. 4073 - 4083, 2014. doi: 10.1109/TIE.2013.2286565.

[9] C. Vázquez, J. Collado, and L. Fridman, "Control of a parametrically excited crane: A vector Lyapunov approach," IEEE Transactions on Control Systems Technology, vol. 21, no. 6, pp. 2332 - 2340, 2013. doi: 10.1109/TCST.2012.2233739.

[10] H.-H. Lee, "A new design approach for the antiswing trajectory control of overhead cranes with high-speed hoisting," International Journal of Control, vol. 77, no. 10, pp. 931-940, 2004. doi: $10.1080 / 00207170412331270550$.

[11] A. Levant, "Higher-order sliding modes, differentiation and output-feedback control," International Journal of Control, vol. 76, no. 9-10, pp. 924-941, 2003. doi: 10.1080/0020717031000099029.

[12] S. Mobayen and F. Tchier, "A novel robust adaptive second-order sliding mode tracking control technique for uncertain dynamical systems with matched and unmatched disturbances," International Journal of Control, Automation and Systems, vol. 15, pp. 1097-1106, jun 2017. doi: 10.1007/s12555-015-0477-1.

[13] Q. P. Ha, M. T. Nguyen, J. Li, and N. M. Kwok, "Smart Structures with Current-Driven MR Dampers: Modelling and Second-Order Sliding Mode Control," IEEE/ASME Transactions on Mechatronics, vol. 18, no. 6, pp. 17021711, 2013. doi: 10.1109/TMECH.2013.2280282.

[14] G. Bartolini, A. Pisano, and E. Usai, "Second-order sliding-mode control of container cranes," Automatica, vol. 38, no. 10, pp. 1783-1790, 2002. doi: 10.1016/S00051098.

[15] R. M. T. Raja Ismail, N. D. That, and Q. P. Ha, "Modelling and robust trajectory following for offshore container crane systems," Automation in Construction, vol. 59, pp. 179187, 2015. doi: 10.1016/j.autcon.2015.05.003.

[16] S. Moon, W. Lee, and S. Lee, "Adaptive sliding mode control of overhead cranes with varying cable length," Journal of Mechanical Science and Technology, vol. 27, no. 3, pp. 885-893, 2013. doi: 10.1007/s12206-013-0204-x.

[17] Q. Ngo and K. Hong, "Adaptive sliding mode control of container cranes," IET control theory and applications, vol. 6, no. 5, pp. 662-668, 2012. doi: 10.1049/ietcta.2010.0764.

[18] X. Yu and M. Zhihong, "Multi-input uncertain linear systems with terminal sliding-mode control," Automatica, vol. 34, no. 3, pp. 389-392, 1998. doi: 10.1016/S00051098.
[19] S. Yu, X. Yu, B. Shirinzadeh, and Z. Man, "Continuous finite-time control for robotic manipulators with terminal sliding mode," Automatica, vol. 41, no. 11, pp. 1957-1964, 2005. doi: 10.1016/j.automatica.2005.07.001.

[20] S. Wu and J. Zhang, "A terminal sliding mode observer based robust backstepping sensorless speed control for interior permanent magnet synchronous motor," International Journal of Control, Automation and Systems, vol. 16, no. 6, pp. 2743-2753, 2018. doi: 10.1007/s12555-0170806-7.

[21] X. Yu and M. Zhihong, "Fast terminal sliding-mode control design for nonlinear dynamical systems," IEEE Transaction on Circuits and Systems, vol. 49, no. 2, pp. 261-264, 2002. doi: 10.1109/81.983876.

[22] D. Zhao, S. Li, and F. Gao, "Finite time position synchronised control for parallel manipulators using fast terminal sliding mode," International Journal of Systems Science, vol. 40, no. 8, pp. 829-843, 2009. doi: 10.1080/00207720902961022.

[23] W. Wang, J. Yi, D. Zhao, and D. Liu, "Design of a stable sliding-mode controller for a class of second-order underactuated systems," IEE Proceedings-Control Theory and Applications, vol. 151, no. 6, pp. 683 - 690, 2004. doi: 10.1049/ip-cta:20040902.

[24] H. Olsson, K. J. Astrom, C. C. De Wit, M. Gafvert, and P. Lischinsky, "Friction models and friction compensation," Eur. J. Control, vol. 4, no. 3, pp. 176-195, 1998. doi: 10.1016/S0947-3580(98)70113-X.

[25] W.-F. Xie, "Sliding-mode-observer-based adaptive control for servo actuator with friction," IEEE Transactions on Industrial Electronics, vol. 54, no. 3, pp. 1517-1527, 2007. doi: 10.1109/TIE.2007.894718.

[26] M. Zak, "Terminal attractors for addressable memory in neural networks," Physics Letters A, vol. 133, no. 1-2, pp. 18-22, 1988. doi: 10.1109/9.489220.

[27] K.-B. Park and J.-J. Lee, "Comments on" A robust MIMO terminal sliding mode control scheme for rigid robotic manipulators"," IEEE Transactions on Automatic Control, vol. 41, no. 5, pp. 761-762, 1996. doi: 10.1109/9.489220.

[28] A. M. Singh, V. T. Hoang, and Q. P. Ha, "Fast terminal sliding mode control for gantry cranes," 33rd International Symposium on Automation and Robotics in Construction (ISARC 2016), pp. 437-443, 2016. doi: 10.22260/ISARC2016/0053.

[29] C.-L. Hwang, C.-C. Chiang, and Y.-W. Yeh, "Adaptive Fuzzy Hierarchical Sliding-Mode Control for the Trajectory Tracking of Uncertain Underactuated Nonlinear Dynamic Systems," IEEE Transactions on Fuzzy Systems, vol. 22, no. 2, pp. 286-299, 2015. doi: 10.1109/TFUZZ.2013.2253106.

[30] B. L. Ma, "Comment 'Design of a stable sliding-mode controller for a class of second-order underactuated systems'," IET Control Theory and Applications, vol. 1, no. 4, pp. 1186-1187, 2007. doi: 10.1049/iet-cta.2009.0345.

[31] K. B. Park, "Comments on 'design of a stable sliding-mode controller for a class of second-order underactuated Systems'," IET control theory and applications, vol. 6, no. 8, pp. 1153-1153, 2012. doi: 10.1049/iet-cta.2009.0345. 
[32] J.-A. Escareno, M. Rakotondrabe, and D. Habineza, "Backstepping-based robust-adaptive control of a nonlinear 2-DOF piezoactuator," Control Engineering Practice, vol. 41, pp. 57-71, 2015. doi: 10.1016/j.conengprac.2015.04.007.

[33] D. Qian and J. Yi, Hierarchical sliding mode control for under-actuated cranes. Springer, 2016. doi: 10.1007/9783-662-48417-3.

[34] B. Yao and M. Tomizuka, "Smooth robust adaptive sliding mode control of manipulators with guaranteed transient performance," Journal of dynamic systems, measurement, and control, vol. 118, no. 4, pp. 764-775, 1996. doi: 10.1115/1.2802355.

[35] D. Whitley, "A genetic algorithm tutorial," Statistics and computing, vol. 4, no. 2 , pp. 65-85, 1994 . doi: 10.1007/BF00175354.
[36] R. R. Selmic and F. L. Lewis, "Deadzone compensation in motion control systems using neural networks," IEEE Transactions on Automatic Control, vol. 45, no. 4, pp. 602613, 2000. doi: 10.1109/9.847098.



Ansu Man Singh received his B.E. in Electronics Engineering from Tribhuwan University, Kathmandu, Nepal in 2008, and M.E. degree from Chonbuk National University, South Korea, in 2013. He is currently pursuing a Ph.D. in University of Technology Sydney (UTS). His research interests include nonlinear control, robust control,and system identification. 